\title{
10. GALACTIC X-RAY POLARIMETRY AND HIGH-RESOLUTION X-RAY SPECTROSCOPY
}

\author{
R. NOVICK \\ Columbia Astrophysics Laboratory, Columbia University, New York, N.Y., U.S.A.
}

\begin{abstract}
Stellar X-ray spectroscopy and polarimetry are discussed in terms of the source parameters that can be determined through such studies and in terms of the constraints that these studies will place on theoretical models. The spectroscopic and polarimetric results that have been obtained to date are reviewed. These include the recent discovery of X-ray polarization in the Crab Nebula and the recent evidence for X-ray coronal line emission in the Cygnus Loop. Finally, the properties and predicted performance of a number of satellite-borne spectrometers and polarimeters are presented.
\end{abstract}

\section{Introduction}

While very great progress has been made in X-ray astronomy with very simple observational tools, it is clear that the further development of the subject requires highresolution spectroscopic and polarimetric observations. The photometric methods that have been used have served to reveal the binary nature of a number of compact $\mathrm{X}$-ray sources. Modulation collimator and lunar occultation data have provided very precise positional data. In a number of cases it has been possible to correlate X-ray sources with particular radio and optical objects. Such correlations have enormously expanded our knowledge of these sources and have yielded some important optical spectroscopic observations on a few of them. Since the X-ray, optical, and radio emission regions may not be identical in the various sources, it is clear that direct $\mathrm{X}$-ray spectroscopic and polarimetric observations must be made if we are to understand the physical conditions in the X-ray emitting regions. Also, since some objects seem to have no observable optical counterpart, we must rely entirely on X-ray observations to understand these objects. Thus it is clear that spectroscopic and polarimetric observations are essential for determining the physical conditions in the X-ray sources and for distinguishing between various theoretical models.

In this review we indicate the nature of the information that we hope to obtain about X-ray sources through spectroscopic and polarimetric observations. We discuss instrumental problems, we indicate the present satellite flight schedule for various spectrometers and polarimeters, and finally we review the results that have been obtained to date.

\section{Spectroscopy}

Spectral lines will be observable in all thermal X-ray sources. Knowledge of the width of these lines, their intensities, and possible shifts will be essential for the determination of the temperature, density, abundances, mass motions, and gravitational fields within the emitting region. The spectroscopic techniques that are available include the 
use of proportional counters, solid-state detectors, filters, gratings, and Bragg crystal spectrometers.

Proportional counters are available with large areas and with low background counting rates. Their energy resolution is limited by the relatively high effective ionization energy of the counting gas. Typically $30 \mathrm{eV}$ are required to produce an ion pair in the gas, and this implies a resolution width of $170 \mathrm{eV}$ at $1 \mathrm{keV}$ photon energy.

The most comprehensive development on proportional counters has been accomplished by Boldt and his collaborators of the NASA Goddard Space Flight Center. This group has pioneered in the development of multiwire, multidepth proportional counters in which they have achieved a resolution of $840 \mathrm{eV}$ with the $6-\mathrm{keV}^{55} \mathrm{Fe} \mathrm{X}$-ray line (Bleach et al., 1972). At longer wavelengths, a resolution of $190 \mathrm{eV}$ has been reported for the $282-\mathrm{eV}$ carbon-K line and $170 \mathrm{eV}$ at the $185-\mathrm{eV}$ boron-K line with simple proportional counters operating with pure methane gas (Yentis et al., 1971).

Solid-state detectors require only a few electron volts per ion pair and therefore exhibit much higher resolution than proportional counters. Goulding and Stone (1970) have reviewed the status of the development of solid-state X-ray detectors. At low photon energies, the resolution is limited by preamplifier noise, and the best reported resolution at long wavelengths is $105 \mathrm{eV}$ (Landis et al., 1970). Unfortunately, it seems to be difficult to produce large-area, high-resolution solid-state detectors, and in addition they must be refrigerated to obtain the best resolution. In view of their small size it appears that solid-state detectors can best be used in conjunction with focusing optics. Very dramatic improvements could be made in X-ray astronomy if large-area, highresolution, position-sensitive solid-state detectors could be developed. Among other applications, such a detector could be used at the focus of a true X-ray telescope to serve as an image-forming element with reasonable spectral resolution. One advantage of these detectors over proportional counters for this application is the fact that the detectors are relatively thin; they therefore could be used with very high-resolution telescopes which have a limited depth of focus. This application would require spatial resolution on the order of $0.02 \mathrm{~mm}$.

In May, 1971, Singer (1972), of the Los Alamos Scientific Laboratory, flew three lithium-drifted silicon detectors in a rocket designed to study the spectrum of Sco X-1. Each detector had an area of $1.1 \mathrm{~cm}^{2}$, they were cooled with liquid nitrogen, and the resolution widths, determined by electronic noise, were about $250 \mathrm{eV}$. One detector provided background information. While the final results have not been published, Singer finds that the energy spectrum is flat from about $700 \mathrm{eV}$ to $4 \mathrm{keV}$ with a constant intensity (within $20 \%$ ) of $25 \pm 5 \mathrm{keV} \mathrm{keV}^{-1} \mathrm{~cm}^{-2} \mathrm{~s}^{-1}$. At higher energy, the spectrum falls according to the well-known thermal bremsstrahlung formula with a temperature in the range of about $3 \mathrm{keV}$. Singer finds no evidence for any lines, but the area of his detector is so small that even with the improved resolution, he would not have been able to detect the 7-keV feature suggested by proportional counter experiments (see below). Singer has stated that his data suggest the presence of an absorption feature at the neon absorption edge, but the statistical significance of this feature is not clear. 
Womack and Overbeck (1972), of the Massachusetts Institute of Technology, have also flown a solid-state detector in a balloon, but their results have not yet been published. The X-ray astronomy group of the NASA Goddard Space Flight Center is scheduled to fly a cooled nonimaging solid-state detector at the focal plane of the high-efficiency telescope in the HEAO-C spacecraft.

The theoretical resolution $(\lambda / \Delta \lambda)$ of gratings is equal to the total number of lines in the grating. In practice, the resolution is determined by the width of the entrance and exit slits and by aberrations. Various groups are working on true astigmatic focusing holographic gratings of sufficient size to be useful for nonsolar X-ray astronomy (Pieuchard et al., 1972). Diffraction gratings were successfully used to study the solar corona in the far ultraviolet during the March, 1970, solar eclipse (Speer et al., 1970). The American Science \& Engineering (AS\&E) X-ray astronomy group is scheduled to fly a normal incidence transmission objective grating on the HEAO-C spacecraft. It is expected that the resolution $(\lambda / \Delta \lambda)$ of this grating will be about 50.

True Bragg crystal spectrometers offer a very great promise in stellar X-ray astronomy. In view of the low fluxes from most sources, it is necessary to employ crystal areas of several thousand square centimeters or more. The X-ray astronomy groups at the University of Leicester, at the Los Alamos Scientific Laboratory, and at the Columbia Astrophysics Laboratory have flown large-area spectrometers in sounding rockets to search for the Fe XXV, the $\mathrm{S} X V I$, the $\mathrm{Ne} I \mathrm{IX}$, and the $\mathrm{O}$ VIII lines in Sco $\mathrm{X}-1$.

In the first Leicester flight on March 18, 1970, a lithium fluoride spectrometer with an effective crystal area of $220 \mathrm{~cm}^{2}$ was used to search for the $\left(1 s^{2}{ }^{1} S-1 s 2 p^{1} P\right)$ line of Fe XXV $(6.70 \mathrm{keV})$ in the X-ray spectrum of Sco X-1 (Griffiths et al., 1971). No line was detected, and an upper limit (at the 3- $\sigma$ level of confidence) of $1.2 \times 10^{-9}$ ergs $\mathrm{cm}^{-2} \mathrm{~s}^{-1}$ was established on narrow-line emission from Sco X-1 between $1.83 \AA$ and $1.91 \AA$. The overall spectrum between 4 and $14 \mathrm{keV}$ was observed with a proportional counter, and it was found that the electron temperature was $6 \times 10^{7} \mathrm{~K}$. By combining this result with the above limit on the line flux, it was found that the 3- $\sigma$ upper limit on the equivalent width of any narrow line between 6.51 and $6.77 \mathrm{keV}$ was $50 \mathrm{eV}$. The alignment of the crystals of this spectrometer was improved so that the width of the rocking curve was reduced from $10^{\prime}$ to $5^{\prime}$. This improved spectrometer was flown on March 11, 1971, in another search for the Fe Xxv lines in Sco X-1. Again, no lines were observed, and the 3- $\sigma$ limit on narrow-line emission from $\mathrm{Fe} \mathrm{XXV}$ was reduced to an equivalent width of $25 \mathrm{eV}$ when the source temperature was $5.5 \times 10^{7} \mathrm{~K}$ (Griffiths, 1972).

The Los Alamos Scientific Laboratory flew mica and KAP crystal spectrometers in a rocket to search for the Ne IX and O VIII lines in Sco X-1. Each spectrometer employed two crystal panels each with an area of about $290 \mathrm{~cm}^{2}$. The continuum from Sco X-1 was observed with each spectrometer, but no lines were observed. The authors have not given upper limits on either the line strengths or the equivalent widths of these lines (Argo et al., 1972). 
On April 24, 1970, the Columbia Astrophysics Laboratory flew a 2000-cm² graphite crystal spectrometer in an Aerobee-170 rocket to search for the S XVI line in Sco X-1 (Kestenbaum et al., 1971). Again, the continuum was detected with a temperature of $8 \times 10^{7} \mathrm{~K}$, but no line was observed. The 3- $\sigma$ upper limits on line strength and equivalent width were 0.08 photons $\mathrm{cm}^{-2} \mathrm{~s}^{-1}$ and $6.7 \mathrm{eV}$, respectively. In a more recent Aerobee-170 rocket flight on March 22, 1972, a lithium-fluoride spectrometer with $3400-\mathrm{cm}^{2}$ crystal area was flown to search for the Fe Xxv lines in Sco X-1. No lines were observed, and it was shown that at the 3- $\sigma$ level the equivalent width of the Fe XXV lines was $3 \mathrm{eV}$ or less (Stockman et al., 1972).

It is generally agreed that the absence of sharp lines in Sco X-1 results from severe broadening in this dense object. The first estimate of these effects was made by Angel (1969a) who showed that at a temperature of $5 \times 10^{7} \mathrm{~K}$ and a density of $10^{16}$ electrons $\mathrm{cm}^{-3}$, suggested by the infrared observations, the line strengths will be decreased by a factor of about 16 by electron scattering. These calculations have been considerably refined and extended by Loh and Garmire (1971) and by Felten et al., (1972). These authors include the effects of photoionization by the intense X-ray flux in Sco X-1 and the effect of trapping on the resonance lines. They show that at a temperature of $7 \times 10^{7} \mathrm{~K}$ and an optical depth of 5 for electron scattering, the equivalent width of the core of the $\mathrm{S} X V I \mathrm{~L} \alpha$ line of $2.62 \mathrm{keV}$ is $0.32 \mathrm{eV}$. In the case of the Fe XxV resonance line at a temperature of $5 \times 10^{7} \mathrm{~K}$ and an optical depth for scattering of 5 , the predicted equivalent width of the line core is $1.9 \mathrm{eV}$. Under the same conditions, the sum of the equivalent widths of the Fe XXV forbidden and intercombination lines is predicted to be $34 \mathrm{eV}$. Thus, the observations indicate that either the temperature or the density is higher than the values assumed above. For example, according to Felten et al. (1972), if the temperature were $7 \times 10^{7} \mathrm{~K}$ and the optical depth were 10 , then the predicted equivalent width of the $\mathrm{Fe} X X V$ resonance and intercombination lines would be about $3 \mathrm{eV}$, consistent with the observations. Alternatively, the temperature and density may have lower values suggested above, but there may be appreciable mass motion and turbulence in the source. Such motion would, of course, broaden the line core remaining after scattering. Mass motion is expected if the radiation arises in an accretion disk surrounding a dense stellar object. In any case it is clear that any further observations of Sco X-1 require long-term satellite observations. As an example of the power of satellite spectrometers, we note that the Bragg crystal spectrometer on HEAO-B (see below) will have sufficient sensitivity to detect the Fe XXV and Fe XXVI lines in Sco X-1 in one day at the 3- $\sigma$ level if the equivalent width of the core is $0.2 \mathrm{eV}$ or more. In addition, this spectrometer will have sufficient sensitivity to reveal the $O$ vil coronal lines in Alpha Centauri at the 3- $\sigma$ level in five days if they have the same strength as those observed on the Sun. The detection of the lines in Sco X-1 and other compact sources is essential for determining the density, temperature, and velocity distribution in the $\mathrm{X}$-ray emitting region.

In addition to the Bragg crystal and solid-state detector studies of Sco X-1 discussed above, numerous observations have been made of this object with proportional counters. Four groups report weak, broad features in the neighborhood of the 7-keV 
iron lines. The U.S. Naval Research Laboratory reports a 3- $\sigma$ upper limit on iron line emission from Sco X-1 with an equivalent width of about $50 \mathrm{eV}$ at a temperature of $5.5 \times 10^{7} \mathrm{~K}$ (Meekins et al., 1969). The NASA Goddard Space Flight Center reported a 3.25- $\sigma$ iron line feature with an equivalent width of about $50 \mathrm{eV}$ and a temperature of $6 \times 10^{7} \mathrm{~K}$ (Holt et al., 1969, 1970). The University of Leicester reported a 5- $\sigma$ upper limit on the iron line with an equivalent width of $100 \mathrm{eV}$ at a temperature of $5.5 \times 10^{7} \mathrm{~K}$ (Griffiths, 1972). The Lockheed group reported an observation of iron line emission with an equivalent width of $240 \pm 140 \mathrm{eV}$ at a temperature of $5.5 \times 10^{7} \mathrm{~K}$ (Acton et al., 1970). In the case of the Lockheed experiment, it should be noted that the detector used had a very thick window that caused considerable curvature in the observed spectrum at the position of the iron line. Ignoring the Lockheed result, we find that the equivalent width of the iron lines in Sco X-1 as observed with proportional counters is $100 \mathrm{eV}$ or less. Since the resolution width of a proportional counter of $7 \mathrm{keV}$ is about $1 \mathrm{keV}$, these results cannot be directly compared with the estimates of Felten et al. (1972). As noted above, these authors estimated the equivalent width of the cores of the Fe XXV and S XVI lines as well as the equivalent width of the total line. Since the total line energy is spread over several keV (Angel, 1969a), it will be necessary to make detailed predictions of the line shape before making a comparison with theory. As yet no detailed line-shape or continuum-shape predictions have been made for compact $\mathrm{X}$-ray sources.

The HEAO-B satellite will contain two stellar Bragg crystal X-ray spectrometers, one to cover the 1 to $8 \mathrm{keV}$ region and the other to cover the 0.4 to $2 \mathrm{keV}$ region. This will be the first satellite-borne spectrometer to observe the region below $1 \mathrm{keV}$. The higher-energy spectrometer will contain flat crystals for high resolution such as germanium with subarc-minute rocking curves and crystals for high integrated reflectivity such as graphite to observe broad lines and the detailed shape of continua. Mounted on the back of the high-energy crystal panel will be another crystal such as PET. The panel can be rotated to expose either the PET or the germanium and graphite crystals. The lower-energy spectrometer will use flat crystals of RAP and a thin window counter as detector. It is hoped to collect the radiation reflected by the crystal in paraboloidal mirrors and to focus the radiation into very small detectors in order to reduce the background rate. It is also hoped to put an array of grazing-incidence gratings back to back to the RAP to be able to observe in the energy range 0.1 to $0.5 \mathrm{keV}$. The area of each crystal panel will be $2300 \mathrm{~cm}^{2}$. The orientation of both the crystal panels and the detectors can be controlled in orbit in order to perform a Bragg scan. Sources can be observed for several days if necessary.

In addition to HEAO-B, the OSO-I, ANS, UK-5, and the HEAO-C spacecraft will also contain stellar Bragg crystal X-ray spectrometers. The OSO-I instrument will contain a large-area mosaic crystal spectrometer that will be useful for determining the exact shape of the continua from a number of sources, and in addition, it will provide a complete spectral survey of all of the presently known sources. The OSO-I spectrometer consists of two $970-\mathrm{cm}^{2}$ graphite crystal panels that view the X-ray sky through the edge of the satellite wheel compartment. The angle between the panels is 
$40^{\circ}$ and located between them is a two-sided proportional counter. The Bragg scan from $9^{\circ}$ to $84^{\circ}$ ( 11.8 to $1.86 \mathrm{keV}$ ) is accomplished by the rotation of the satellite wheel compartment.

The ANS instrument consists of two $65-\mathrm{cm}^{2}$ EDDT or PET crystal panels oriented at about $45^{\circ}$ to the viewing direction. One crystal is adjusted to detect the Si XIII lines and the other the Si XIV line. The spacecraft can be oriented so that a source can be studied for about one-half day. The UK-5 spectrometer will employ graphite and lithium hydride crystal panels. Each panel has an area of about $200 \mathrm{~cm}^{2}$. The Bragg angle is adjustable in flight from $25^{\circ}$ to $65^{\circ}$ and can be set at $45^{\circ}$ for polarimetry (see below). The spectral range of the instrument extends from 2 to $8 \mathrm{keV}$. The viewing direction of the spectrometer is parallel to the spacecraft spin axis so that sources may be observed for extended periods of time. The HEAO-C spectrometers employ very high-resolution, curved crystals at the focus of the high-resolution ( $\left.1^{\prime \prime}\right)$ telescope. These instruments will permit detailed studies of the line shapes and shifts.

Angel and Weisskopf (1970) have proposed and tested a novel Bragg objective crystal spectrometer that appears to have a great promise. Briefly, the instrument consists of a large-area crystal mounted in front of an X-ray telescope. The angle between the telescope axis and the crystal plane and the angle between the crystal plane and the line of sight to the X-ray star are chosen to satisfy the Bragg condition for a particular X-ray line or multiplet. A spectral line appears in the focal plane of the telescope as an arc of a large diameter circle. The resolution of the instrument is determined by the diffraction width of the individual domains in the crystal panel, and the spectral range is determined by the mosaic spread in the crystal and the field of view of the telescope. Since the entire aperture of the telescope can be illuminated by the diffracted beam, the instrument has very high sensitivity. A schematic diagram of this spectrometer is shown in Figure 1, and in Figure 2 we show the rhodium $L \alpha_{1}$ and $L \alpha_{2}$ lines as observed in the laboratory with a small grazing incidence telescope and an objective mosaic graphite crystal. The $3.5-\mathrm{eV}$ linewidth observed here is determined by the natural width of the rhodium lines and is in excellent agreement with the $2.75-\mathrm{eV}$ width obtained with a very high-quality calcite Bragg crystal spectrometer. The energy and Bragg-angle separation of the $\mathrm{L} \alpha_{1}$ and $\mathrm{L} \alpha_{2}$ lines are $4.69 \mathrm{eV}$ and $5.5^{\prime}$, respectively. The mosaic spread of the crystal used in this experiment was $24^{\prime}$. The fact that the $\mathrm{L} \alpha_{1}$ and $\mathrm{L} \alpha_{2}$ lines could be clearly resolved shows that the resolution of the objective crystal spectrometer is determined by the diffraction width of the individual domains rather than the mosaic spread of the crystal.

The only object besides Sco X-1 which has been subject to detailed spectroscopic study is the Cygnus Loop. It is believed that this is a supernova remnant and that the emitting region is a low-density, high-temperature plasma. If this is correct, then coronal lines should be observable in both the X-ray and visible bands. In a collaborative experiment between AS \&E and Columbia, the Cygnus Loop was observed with a one-dimensional focusing collector and thin window proportional counter (Gorenstein et al., 1971). The data rule out a power-law spectrum and strongly suggest a thermal bremsstrahlung spectrum with $T=4.3 \times 10^{6} \mathrm{~K}$ and a line at $0.65 \mathrm{keV}$ in the 




Fig. 1. Schematic diagram of the Bragg objective crystal spectrometer.

neighborhood of the expected coronal O VII and O viII lines. Tucker (1971) reinterpreted these data and suggested that the temperature might be $2 \times 10^{6} \mathrm{~K}$. The interpretation of the line depends on our knowledge of the reflection efficiency of the focusing collector since this instrument employed a chromium surface which has a strong absorption feature close to the oxygen lines. The reflection efficiency of the mirror was measured at $10 \AA$ and $44 \AA$ and found to be in good agreement with the results of Ershov et al. (1967). Thus there was no reason to believe that the assumed reflection efficiency in the neighborhood of $0.65 \mathrm{keV}$ is incorrect. Recently Bleeker et al. (1972) reported on a very careful proportional counter study of the Cygnus Loop. They confirmed the thermal spectrum with $T=2.7 \times 10^{6} \mathrm{~K}$ but found that they were 


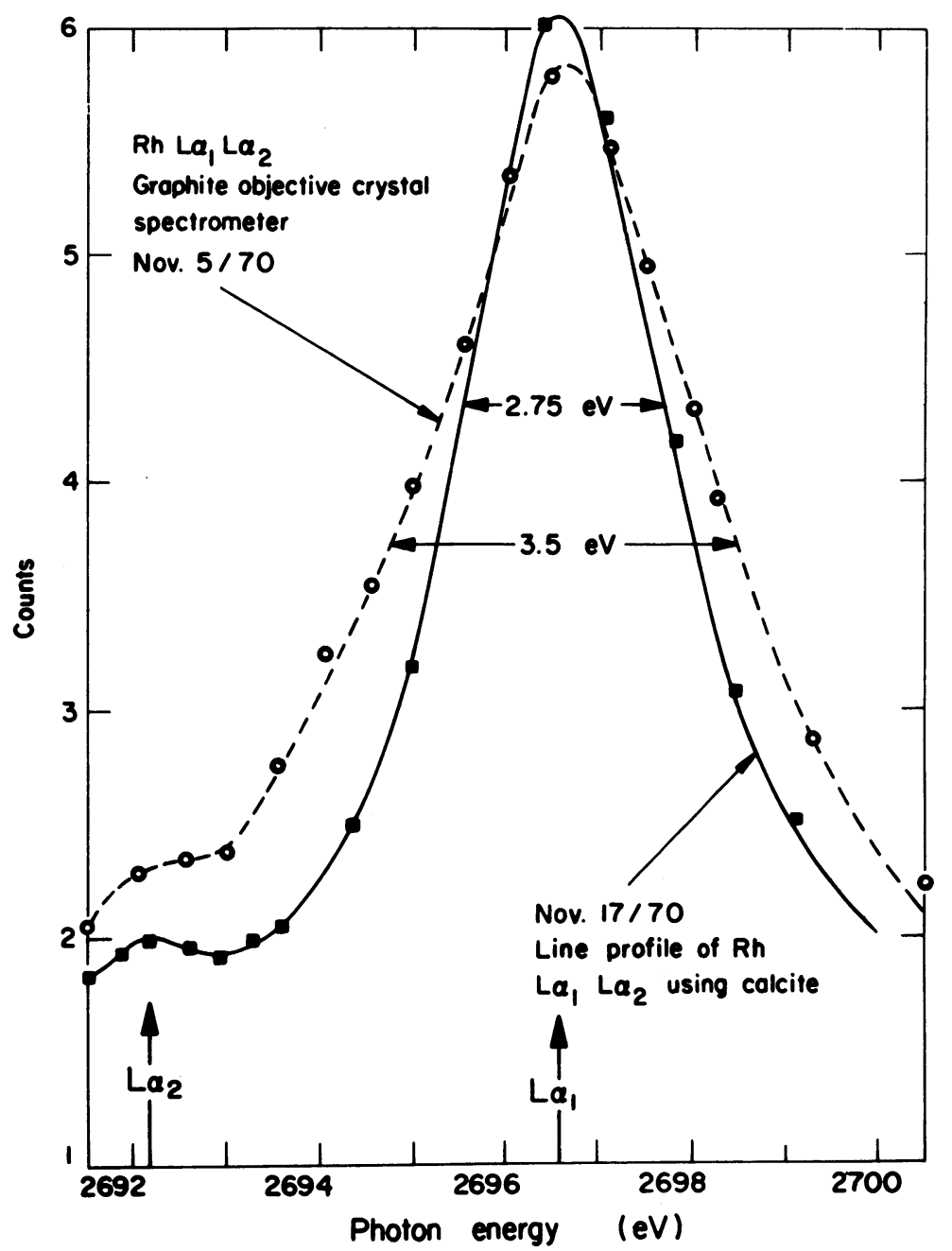

Fig. 2. Rhodium $L \alpha_{1}$ and $L \alpha_{2}$ lines observed with a small mosaic graphite objective crystal spectrometer and with a conventional high-resolution calcite crystal spectrometer.

not required to assume the existence of X-ray lines to fit their data. More recently, Stevens et al. (1972) made a study of this object with proportional counters and balanced absorption filters. One of the filters consisted of a gas cell that was filled with oxygen during the rocket flight. The other was a thin Teflon sheet that provided the fluorine absorption edge. This experiment has provided very strong evidence for the existence of the oxygen lines. Thus, it appears that the Cygnus Loop is the first nonsolar object to exhibit X-ray lines.

Kurtz et al. (1972) have made extensive searches for the optical coronal Fe XIV line at $5303 \AA$ in the Cygnus Loop, but so far they have failed to detect the lines. Using a filter with $3-\AA$ bandwidth, they have shown that the $\lambda 5303$ flux from the Cygnus 
Loop is less than $5 \times 10^{-9}$ ergs $\mathrm{cm}^{-2}$ sterad $^{-1} \mathrm{~s}^{-1}$. This is an order of magnitude less than the predicted total flux for a plasma temperature of $2.7 \times 10^{6} \mathrm{~K}$. This negative result is taken to indicate either broadening of the line profile by mass motion or a higher temperature in the Loop. Clearly, it is essential not only to detect the X-ray and optical lines but also to determine their intensity and profile. This information will provide a direct measure of the temperature and velocity distribution within the Loop and will certainly help to clarify the difference between the $110 \mathrm{~km} \mathrm{~s}^{-1}$ velocity observed in the filaments and the $430 \mathrm{~km} \mathrm{~s}^{-1}$ shock velocity required by the X-ray observations (Woltjer, 1972).

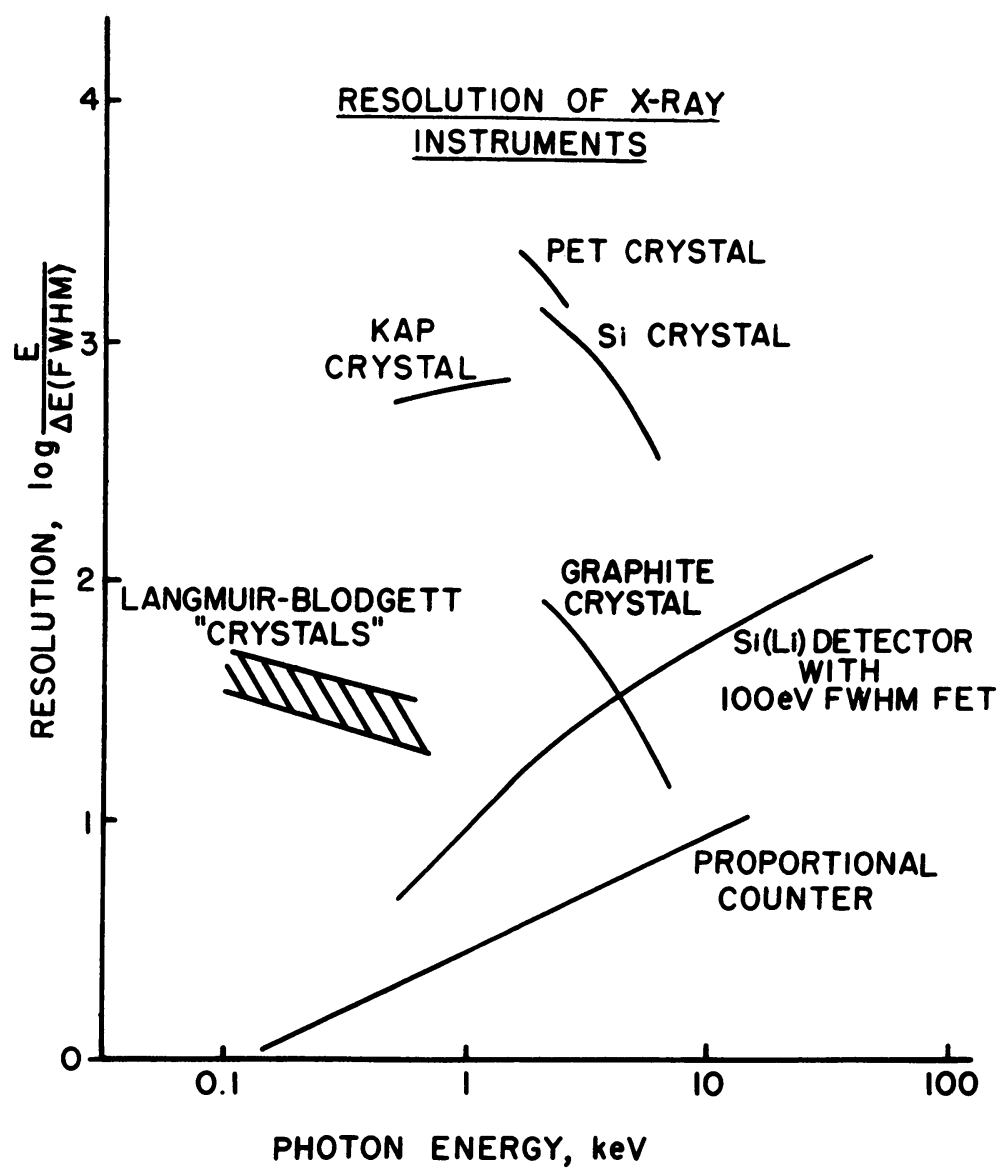

Fig. 3. Resolution of $X$-ray instruments.

In this section we have indicated the present status of X-ray spectroscopy, particularly in regard to Sco X-1 and the Cygnus Loop. Also, we have indicated the instrumental developments, and we have briefly indicated the forthcoming satellite $\mathrm{X}$-ray spectroscopy experiments. In Figure 3, we briefly summarize the resolution of various types of spectrometers. 


\section{Polarimetry}

$\mathrm{X}$-ray polarization is expected in any source that is either nonthermal or aspherical. In the case of synchrotron emission, it is well known that linear polarization is observed, that it is independent of energy, and that the polarization pattern within an extended source provides a map of the magnetic field. In the case of a dense aspherical thermal source, polarization of a few percent is expected if there is strong scattering within the source (Angel, 1969b). Recently Pringle and Rees (1972) suggested a model for the pulsating binary X-ray source Cen X-3 in which the emission results from the accretion of matter onto the poles of a rotating magnetic neutron star. If the model is correct, it is expected that the source will exhibit both linear and circular polarization and that the polarization will depend upon the X-ray pulse phase. In this review we will primarily discuss linear polarization, but we note in passing that X-ray circular polarization has been detected by observing the Compton scattering of photons by the polarized $d$ shell electrons in magnetized iron (Goldhaber et al., 1958).

It is, of course, well known that the Crab pulsar NP 0532 exhibits phase-dependent linear polarization in both the radio and optical bands, and it is expected that X-ray polarization will also be observed in this object. In the exploding galaxy $\mathbf{M} 87$, it is known that polarization up to $20 \%$ exists in the visible band in some of the 'knots' in the jet. This fact is taken as evidence of synchrotron emission (Hiltner, 1959). If the $\mathrm{X}$-ray emission occurs by the same process, then we might expect similar polarization. Since the average polarization for the entire galaxy is much lower than the above amount, we can only expect to observe polarization with a nonimaging polarimeter if the X-ray emission is primarily confined to one or two 'knots'. One of the favorite models for the compact X-ray source Sco X-1 ascribes the X-ray emission to a hightemperature accretion disk that surrounds a white dwarf member of a contact binary star system. As yet no evidence has been found for binary motion in this source. If the accretion model is correct, then we might expect a few percent X-ray polarization (Angel, 1969b) due to scattering within the disk. Thus, polarimetry may be useful for establishing the true nature of this and other compact X-ray sources.

Finally, it is noted that, in the impact of monodirectional electrons onto matter, linear polarization is observed and that the sign and magnitude of the polarization depend on the photon energy. At low photon energies, the polarization (electric vector) is perpendicular to the direction of electron flow. At photon energies near the upper end of the bremsstrahlung spectrum, the polarization is parallel to the electron flow. This process is known as linear bremsstrahlung and is believed to account for the X-ray polarization observed by Tindo et al. (1971) in solar flares. It is conceivable that a similar process occurs in the flares observed in Sco X-1 and the other highly variable X-ray sources. In this case it is critical to observe the energy dependence of the polarization with a broad-band polarimeter. It is amusing to speculate that the high-energy nonthermal spectral 'tail' observed in Sco X-1 at energies above $40 \mathrm{keV}$ may be produced by the synchrotron process and may exhibit strong polarization. It is clear from the above discussion that polarimetry is essential for the determination 
of both the structure and X-ray emission mechanisms in a number of X-ray sources.

The techniques that have been used for X-ray polarimetry depend on the angular asymmetry of electron scattering. If the electrons are essentially free (i.e., their binding is small compared to the photon energy), then scattering is observed over a broad energy band, and it has a characteristic dipole form with the axis of the dipole coaligned with the polarization (electric vector) of the incident polarized photon. If the electrons are bound in a regular crystal lattice, than the scattering (Bragg reflection in this case) is restricted to a narrow energy band given by the mosaic spread of the crystal. The scattering observed with a Bragg angle of $45^{\circ}$ is zero if the polarization vector is in the plane of incidence, and it is maximum if it is perpendicular to this plane. In principle, other techniques can be used for polarimetry, but so far none of them have proved as effective as the scattering method. The technique most often considered involves detecting the direction of the primary photoelectron track. Unfortunately, at the energy at which most X-ray sources are most intense (1-20 keV), it is very difficult to detect the direction of an electron track. At energies of $50 \mathrm{keV}$ and higher, this method is probably superior to the scattering method. At much higher energies it will probably be best to determine polarization from the orientation of the plane of the electron pairs formed by a converter in a spark chamber.

All of the X-ray polarization studies that have been made to date have used either Thomson scattering in lithium or beryllium or Bragg reflection at $45^{\circ}$ on mosaic graphite crystals. Mosaic graphite is used for polarimetry since it has the highest integrated reflectivity at an energy $(2.6 \mathrm{keV})$ near the maximum in the spectrum of most X-ray sources. The Thomson-scattering polarimeters provide data over a broad band, set at the lower end (about $5 \mathrm{keV}$ in lithium) by photoelectric absorption in the scattering material and at the upper end by the rapid decrease in flux from the $\mathrm{X}$-ray sources. Since the efficiency of all polarimeters is quite low, it is essential to go to great lengths to reduce the non-X-ray background. In the case of Bragg crystal polarimeters, this may be accomplished in part by mounting the crystals on an arc of a parabolic surface so that the reflected X-rays are focused onto a small detector. This results in a substantial reduction in the size of the detector and yields a commensurate reduction in the non-X-ray background event rate. This type of polarimeter has been discussed in detail elsewhere (Weisskopf et al., 1972). The Thomson-scattering polarimeter has been discussed by Angel et al. (1969), by Wolff et al. (1970), and by Tindo et al. (1970). The Thomson-scattering and graphite polarimeters have been flown in several rocket flights by the X-ray astronomy group at Columbia University. This work has culminated in the detection of X-ray polarization from the Crab Nebula as described below. The Thomson-scattering polarimeters constructed by the group at the Lebedev Institute in Moscow have been flown on Intercosmos 1 and 4. These have been used to study solar flares and, as indicated above, this group has detected strong solar flare X-ray polarization. This work has recently been summarized by Tindo $e t$ al. (1971). Bragg-crystal polarimeters are scheduled to be flown on the UK-5 and OSO-I satellites. The UK-5 instrument also serves as a spectrometer and therefore employs a flat crystal. 
The OSO-I instrument uses a parabolic array of mosaic graphite crystals. Two complete polarimeters will be installed on the spacecraft, and their axes will be roughly orthogonal so as to provide both of the Stokes linear polarization parameters simultaneously. The OSO-I instrument is mounted in the bottom of the wheel section of the OSO vehicle with the polarimeter axis parallel to the spin axis of the wheel.

Sources will be selected by pointing the spin axis toward the source; under normal flight conditions, it will be possible to observe a source for several days. The wheel rotation, of course, provides the desired rotation of the polarimeter to help eliminate spurious systematic effects. The predicted performance of the instrument in both first order, $2.6 \mathrm{keV}$, and second order, $5.2 \mathrm{keV}$, for several interesting sources is shown in Table I.

\section{TABLE I}

Percent minimum detectable polarization at $3-\sigma, 99 \%$ confidence level

\begin{tabular}{lllcl}
\hline Source & $\begin{array}{l}\text { Observing } \\
\text { time (days) }\end{array}$ & $\begin{array}{l}2.6 \mathrm{keV} \\
\text { only }(\%)\end{array}$ & $\begin{array}{l}5.2 \mathrm{keV} \\
\text { only (\%) }\end{array}$ & $\begin{array}{l}\text { Combined } \\
\text { result }(\%)\end{array}$ \\
\hline Crab Nebula (Tau X-1) & 1 & 1.8 & 3.8 & 1.7 \\
Sco X-1 & 1 & 0.59 & 1.1 & 0.52 \\
Cyg X-1 & 1 & 4.4 & 9.0 & 4.0 \\
M 87 (Vir X-1) & 1 & 7.6 & 18 & 7.3 \\
Nor X-2 & 1 & 2.4 & 3.9 & 2.0 \\
Sco X-2 & 1 & 2.7 & 3.8 & 2.2 \\
$\begin{array}{l}\text { Crab Primary Pulse } \\
\text { Cen X-3 (each of four } 0.25-\mathrm{s}\end{array}$ & 6 & 7.0 & 18 & 7.0 \\
$\quad$ bins within pulse) & 6 & 17 & 6.4 & 6.1 \\
\hline
\end{tabular}

It has been proposed to fly a focal plane Bragg crystal polarimeter on the HEAO-C spacecraft. The instrument will be located at the focus of the high-efficiency lens, and it can be used for polarization mapping of extended sources.

The nonsolar X-ray polarization observations that have been made so far have been restricted to Sco X-I and the Crab Nebula. It has been shown that polarization of Sco X-1 is less than $20 \%$ (Angel et al., 1969). This result is entirely consistent with the view that Sco X-1 is a thermal X-ray source. The determination of whether or not Sco X-1 has a small polarization that might arise from scattering within an accretion disk must await the flights of UK-5 and OSO-I. As indicated above, two rocket experiments that have been performed on the Crab Nebula by Wolff et al.(1970) and Novick et al. (1972) have demonstrated that the X-ray emission from this object is polarized. The magnitude and position angle of the polarization are $(15.4 \pm 5.2) \%$ and $(156$ $\pm 10)^{\circ}$, respectively (Novick et al., 1972). This is to be compared to the polarization in the optical of $14 \%$ at a position angle of $154^{\circ}$ when averaged over the X-ray emitting region, which, for the present purpose, is assumed to be $1^{\prime}$ in radius. The equality of the observed X-ray and optical polarizations and the fact that the spectrum can be represented by a power law confirm the view that the synchrotron process is responsible for the X-ray emission. No X-ray polarization would have been observed if the thermal model of Sartori and Morrison (1967) obtained. Thus, the Crab Nebula is the 
first X-ray object for which we have confirmed emission mechanism. This observation poses new problems for pulsar theories since it implies that the synchrotron electron spectrum extends to $6 \times 10^{4} \mathrm{GeV}$ if the Nebular magnetic field is taken as $10^{-4} \mathrm{G}$. Since the radiative lifetime for such electrons is a fraction of a year, they must either be continuously generated by the pulsar, or they must be continuously accelerated by magnetic dipole radiation from the pulsar (Gunn and Ostriker, 1971; Rees, 1971) or by some other presently unknown process. Continuous injection of $6 \times 10^{4} \mathrm{GeV}$ electrons appears to be impossible since in the high magnetic field $\left(10^{12} \mathrm{G}\right)$ of the pulsar the electrons will lose their energy by either synchrotron or curvature radiation before they escape from the immediate neighborhood of the pulsar (Ruderman, 1972). Continuous acceleration by magnetic dipole radiation seems to be ruled out by the fact that Landstreet and Angel (1970) failed to find the circular polarization in the optical band that had been predicted by Rees (1971) for this process. Thus, it appears that we must look elsewhere for either an injection or acceleration mechanism.

From the above discussion, it is clear that both spectroscopy and polarimetry are destined to play an important role in X-ray astronomy. However, most of the critically important results must wait until the various satellite instruments are in orbit.

\section{Acknowledgements}

The author wishes to thank Dr B. Woodgate for many helpful discussions about $\mathrm{X}$-ray spectroscopy and for having prepared Figure 3. The author is indebted to Dr P. Landecker for having evaluated the OSO-I polarimeter sensitivity given in Table I and to Dr M. C. Weisskopf for having made many helpful comments on this manuscript. Finally, he wishes to acknowledge the enthusiastic work of $\mathrm{Mr} \mathrm{H}$. Helava on the objective crystal spectrometer. This work was supported by the National Aeronautics and Space Administration under grants NGR-33-008-102 and NGR-33-008-125. This is Columbia Astrophysics Laboratory Contribution No. 67.

\section{References}

Acton, L. W., Catura, R. C., Culhane, J. L., and Fisher, P. C.: 1970, Astrophys. J. Letters 161, L175.

Angel, J. R. P.: 1969a, Nature 224, 160.

Angel, J. R. P.: 1969b, Astrophys. J. 158, 219.

Angel, J. R. P., Novick, R., Vanden Bout, P., and Wolff, R.: 1969, Phys. Rev. Letters 22, 861. Angel, J. R. P. and Weisskopf, M. C.: 1970, Astron. J. 75, 231.

Argo, H., Evans, D., Bergey, J., and Liefeld, R.: 1972, private communication.

Bleach, R. D., Boldt, E. A., Holt, S. S., Schwartz, D. A., and Serlemitsos, P. J.: 1972, Astrophys. J. 171, 51 .

Bleeker, J. A. M., Deerenberg, A. J. M., Yamashita, K., Hayakawa, S., and Tanaka, Y.: 1972, 'Soft X-ray Spectra of the Cygnus Loop and Cyg X-2 in the Energy Range 0.16-6.7 keV', submitted for publication.

Ershov, O. A., Brytov, I. A., and Lukirskii, A. P.: 1967, Opt. Spectrosc. (U.S.S.R.) 22, 66.

Felten, J. E., Rees, M. J., and Adams, T. F.: 1972, 'Transfer Effects on X-Ray Lines in Optically Thick Celestial Sources', submitted for publication.

Goldhaber, M., Grodzins, L., and Sunyar, A. W.: 1958, Phys. Rev. 109, 1015. 
Gorenstein, P., Harris, B., Gursky, H., Giacconi, R., Novick, R., and Vanden Bout, P.: 1971, Science 172, 369.

Goulding, F. S. and Stone, Y.: 1970, Science 170, 280.

Griffiths, R. E.: 1972, 'A Further High-Resolution Search for Fe xxv Line Emission from Scorpius X-1', Astron. Astrophys. 21, 97.

Griffiths, R. E., Cooke, B. A., and Pounds, K. A.: 1971, Nature Phys. Sci. 229, 175; corrigendum ibid., 231, 136.

Gunn, J. E. and Ostriker, J. P.: 1971, Astrophys. J. 165, 523.

Hiltner, W. A.: 1959, Astrophys. J. 130, 340.

Holt, S. S., Boldt, E. A., and Serlemitsos, P. J.: 1969, Astrophys. J. Letters 158, L155.

Holt, S. S., Boldt, E. A., and Serlemitsos, P. J.: 1970, in L. Gratton (ed.), 'Non-Solar X- and GammaRay Astronomy', IAU Symp. 37, 138.

Kestenbaum, H., Angel, J. R. P., and Novick, R.: 1971, Astrophys. J. Letters 164, L87.

Kurtz, D. W., Vanden Bout, P. A., and Angel, J. R. P.: 1972, 'Search for Coronal Line Emission from the Cygnus Loop', submitted for publication.

Landis, D. A., Goulding, F. S., and Jakpevic, P. M.: 1970, Nuci. Instrum. Meth. (Netherlands) 87, 211.

Landstreet, J. D. and Angel, J. R. P.: 1971, Nature 230, 103.

Loh, E. D. and Garmire, G. P.: 1971, Astrophys. J. 166, 301; erratum, ibid., 169, 447.

Meekins, J. F., Henry, R. C., Fritz, G., Friedman, H., and Byram, E. T.: 1969, Astrophys. J. 157, 197; Astrophys. J. Letters 156, L33.

Novick, R., Weisskopf, M. C., Berthelsdorf, R., Linke, R., and Wolff, R. S.: 1972, Astrophys. J. Letters 174, L1.

Pieuchard, G., Flamand, J., and Laude, J. P.: 1972, 'Recent Developments in the Field of HoloGratings', April 21, Instititute of Physics, Spectroscopy Group and Optical Group, Meeting on Diffraction Gratings, Imperial College, London, England, unpublished.

Pringle, J. E. and Rees, M. J.: 1972, Astron. Astrophys., to be published.

Rees, M. J.: 1971, Nature Phys. Sci. 230, 55.

Ruderman, M.: 1972, private communication.

Sartori, L. and Morrison, P.: 1967, Astrophys. J. 150, 385.

Singer, S.: 1972, private communication.

Speer, R. J., Garton, W. R. S., Goldberg, L., Parkinson, W. H., Reeves, E. M., Morgan, J. F., Nicholls, R. W., Jones, T. J. L., Paxton, H. J. B., Shenton, D. B., and Wilson, R.: 1970, Nature 226, 249.

Stevens, J., Garmire, G., and Riegler, G.: 1972, private communication.

Stockman, H. S., Jr., Angel, J. R. P., Novick, R., and Woodgate, B. E.: 1972, to be submitted for publication.

Tindo, I. P., Shuryghin, A. I., Ivanov, V. D., Vasiljev, V. A., Godrnov, D. A., and Komjak, N. I.: 1970, Kratkie Soobshchenia Po Fisike (U.S.S.R.), No. 7, p. 15.

Tindo, I. P., Valnicek, B., Livshits, M. A., and Ivanov, V. D.: 1971, November 15-19, International Meeting on Solar Activity, Izmiran, U.S.S.R.

Tucker, W.: 1971, Science 172, 372.

Weisskopf, M. C., Berthelsdorf, R., Epstein, G., Linke, R., Mitchell, D., Novick, R., and Wolff, R. S. : 1972, ‘A Graphite Crystal Polarimeter for Stellar X-Ray Astronomy', Rev. Sci. Instr., 43, 967.

Wolff, R. S., Angel, J. R. P., Novick, R., and Vanden Bout, P.: 1970, Astrophys. J. Letters 160, L21.

Woltjer, L.: 1972, 'Supernova Remnants', Ann. Rev. Astron. Astrophys., to be published.

Womack, E. A., Jr. and Overbeck, J. W.: 1972, private communication.

Yentis, D. J., Angel, J. R. P., Mitchell, D., Novick, R., and Vanden Bout, P.: 1971, in F. Labuhn and R. Lüst, (eds.), 'New Techniques in Space Astronomy', IAU Symp. 41, 145. 